\title{
L'expérience VKS2
}

\section{Observation d'une dynamo turbulente et des renversements erratiques du champ magnétique}

Collaboration VKS : CEA - ENS Lyon - ENS Paris - CNRS

francois.daviaud@cea.fr, pinton@ens-lyon.fr, fauve@lps.ens.fr

Nous présentons la première mise en évidence de la génération spontanée d'un champ magnétique sans excitation extérieure (effet dynamo) par un écoulement turbulent de sodium liquide.

Nous rapportons, de plus, la première observation de renversements erratiques du champ magnétique créé par la dynamo, qui évoquent les renversements spontanés du champ magnétique terrestre.

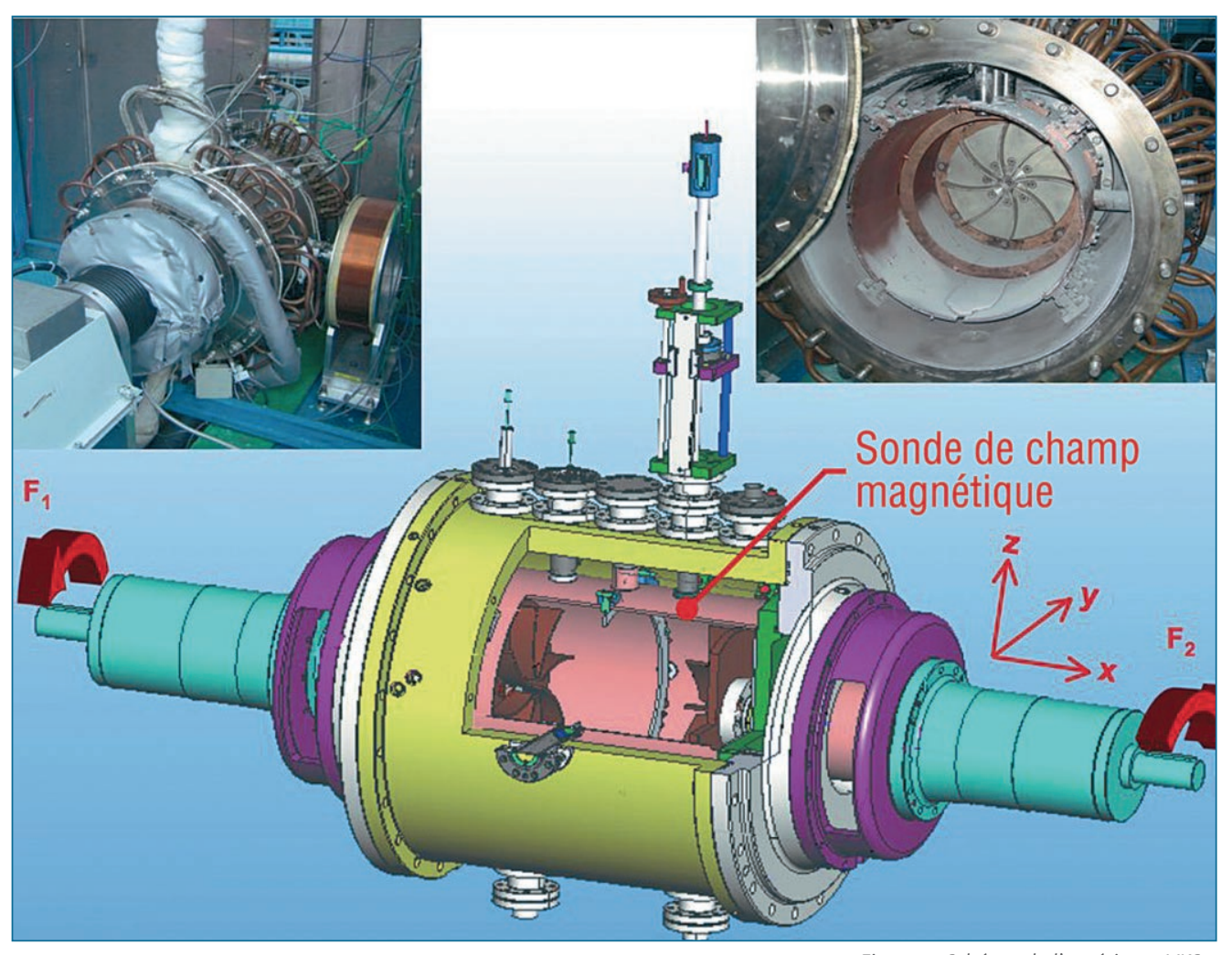

Figure 1. Schéma de l'expérience VKS2. 
Quelle est l'origine du champ magnétique des objets astrophysiques qui nous entourent : planètes, étoiles, galaxies...? Dans le cas du Soleil, Larmor propose en 1919 que ce champ est engendré par effet dynamo (voir encadré p. 16), c'est-à-dire par la création spontanée d'un champ magnétique dans un fluide conducteur en mouvement. Quant au champ magnétique terrestre, il est très probablement créé par le mouvement du fer liquide du noyau. Cet effet est l'analogue des dynamos industrielles (Siemens, 1867) et les équations qui régissent ce phénomène sont connues : équations de Maxwell et loi d'Ohm, équation de NavierStokes. Cependant, si l'on peut mener à bien des calculs analytiques dans le cas d'écoulements simples et indépendants du temps, la prédiction théorique s'avère difficile pour les milieux naturels dans lesquels les mouvements se développent librement et les fluides sont très turbulents. Les simulations numériques ne permettent pas non plus d'atteindre - et ce pour longtemps encore ! - les gammes de paramètres des dynamos naturelles, contrairement aux expériences qui en sont plus proches. Les premières dynamos induites par des écoulements de sodium dans des géométries contraintes reproduisant des solutions analytiques modèles ont été observées en 2000 à Riga et à Karlsruhe. Depuis, plusieurs équipes aux USA, en Russie et en France tentent d'obtenir une dynamo à partir d'écoulements moins contraints, plus proches des systèmes naturels et susceptibles d'engendrer des régimes dynamos plus riches.

Dans le cadre de la collaboration VKS (CEA - ENS Lyon - ENS Paris - CNRS), nous avons réalisé au CEA Cadarache (DEN/DTN) une expérience dans laquelle un écoulement tourbillonnaire, dit de von Karman, est produit par le mouvement de deux turbines tournant en sens inverse dans un cylindre rempli de sodium liquide. L'écoulement est pleinement turbulent avec un nombre de Reynolds (voir encadré p. 16) cinétique $\mathrm{R}_{\mathrm{e}}$ comparable à celui des grandes souffleries aérodynamiques, et il permet d'atteindre des nombres de Reynolds magnétiques $\mathrm{R}_{\mathrm{m}}$ de l'ordre de 50. Les dimensions, les conditions aux limites et la forme des turbines ont fait l'objet de nombreuses études théoriques, numériques et expérimentales (en eau, en gallium et en sodium) [1, 2, 3]. La cuve actuelle fait $600 \mathrm{~mm}$ de long pour un diamètre de $600 \mathrm{~mm}$ et un volume de sodium d'environ 150 litres. Elle comprend une couche de sodium au repos qui entoure l'écoulement, un anneau permettant de stabiliser la couche de cisaillement dans le plan médian et des disques en fer pur (fig. 1). Les mesures de champ magnétique sont réalisées à l'aide de sondes immergées dans l'écoulement.

Dans ces expériences, l'apparition de l'effet dynamo a été marquée par l'apparition spontanée d'un champ magnétique auto-entretenu par le mouvement du fluide, pour une vitesse de rotation des disques supérieure à une vitesse critique (de l'ordre de 1000 tours/minute correspondant à $\mathrm{R}_{\mathrm{m}} \approx 30$, cf. fig. 2b) [4]. Environ $50 \%$ au-dessus du seuil, l'amplitude de ce champ est de l'ordre de 50 Gauss à la frontière de l'écoulement (environ 100 fois la valeur du champ terrestre) et il présente de très fortes fluctuations (fig. 2a). Lorsqu'il est produit, l'effet dynamo est également marqué par une surconsommation d'environ 15\%, mesurée au niveau de l'alimentation des moteurs, 30\% au-dessus du seuil d'instabilité. L'évolution de l'amplitude de l'énergie magnétique locale en fonction de $\mathrm{R}_{\mathrm{m}}$ correspond à une bifurcation légèrement imparfaite autour de $\mathrm{R}_{\mathrm{m}}=30$ et est en bon accord avec une loi d'échelle proposée précédemment pour les grands nombres de Reynolds [5]. Il reste encore à établir dans quelle mesure les fluctuations turbulentes favorisent ou inhibent la dynamo, mais ce résultat montre que les dynamos fluides continuent à opérer en présence de turbulence forte.
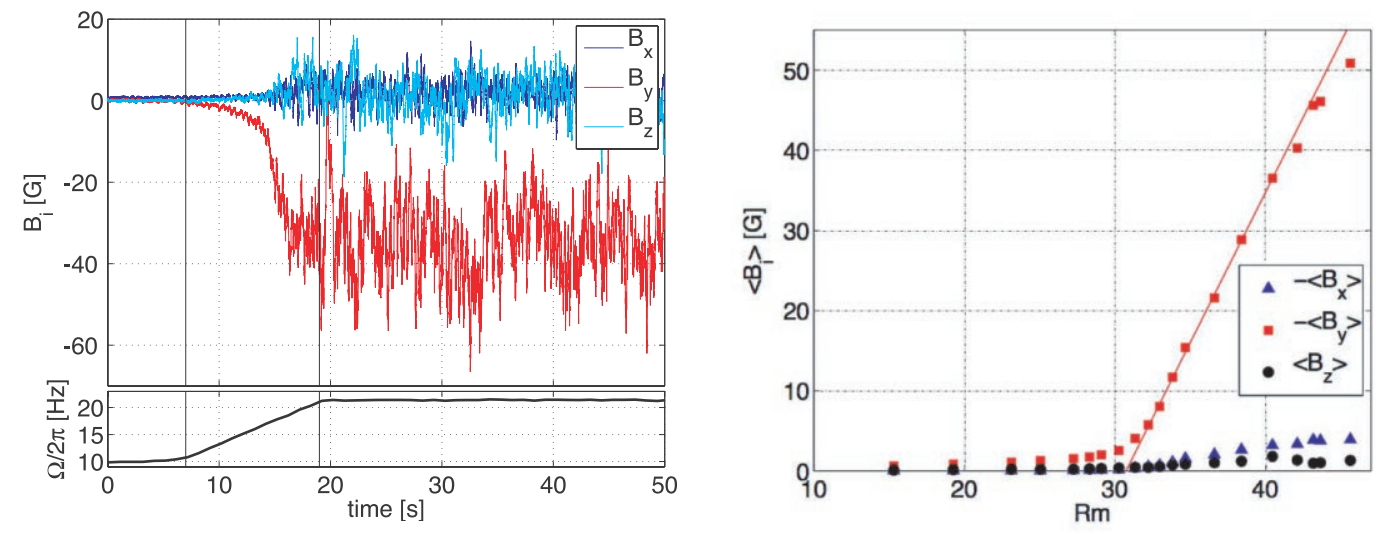

\section{Bibliographie}

[1] R. Volk, et al., Phys. Fluids 18, 085105 (2006).

[2] F. Ravelet, et al.,

Phys. Fluids 17, 117104 (2005)

[3] M. Bourgoin et al.,

Phys. Fluids 14, 2046 (2002).

[4] R. Monchaux et al.,

"Generation of a magnetic field

dynamo action in a turbulent

flow of liquid sodium",

Phys. Rev. Lett. 98, 044502 (2007).

[5] F. Pétrélis et S. Fauve,

Eur. Phys. J. B 22, 273 (2001)

[6] M. Berhanu et al.,

"Magnetic field reversals

in an experimental turbulent

dynamo", Europhys. Lett. 77 (2007),

sous presse.

[7] C. Letellier, Bulletin SFP

154, 10 (mai 2006).

Pour en savoir plus

H.K. Moffatt, Magnetic Field generation in electrically conducting fluids, Cambridge University Press, U.K. (1978)

R. Moreau,

Magnetohydrodynamics, Kluwer Academic Publishers (1990).

F. Daviaud pour l'équipe VKS,

"Expérience VKS : vers la

dynamo turbulente?", Bulletin

SFP 135, 24 (juillet-août 2002).

\section{Liste des participants}

\section{à l'expérience VKS2 :}

M. Berhanu, M. Bourgoin,

A. Chiffaudel, F. Daviaud,

B. Dubruille, S. Fauve, C. Gasquet,

L. Marié, R. Monchaux, N. Mordant,

M. Moulin, P. Odier, F. Pétréli,

J.-F. Pinton, F. Ravelet, R. Volk.

Figure 2. (a) Évolution temporelle des trois composantes du champ magnétique lorsque la vitesse de rotation $F=\Omega / 2 \pi$ est augmentée au-dessus du seuil (contra-rotation exacte). La composante la plus élevée, $B_{y}$, est tangente au cylindre à l'emplacement de la mesure (voir fig. 1) ; (b) évolution des valeurs moyennes des trois composantes du champ magnétique en fonction du nombre de Reynolds magnétique $R_{m}$. L'ajustement linéaire de $B_{y}$ (droite rouge) définit la valeur seuil de $R_{m}$ $R_{m c} \sim 31$. 
Figure 3 : Évolution temporelle présentant les inversions erratiques du champ magnétique lorsque les deux turbines ne tournent pas à la même vitesse $\left(F_{1} \neq F_{2}\right)$. $B_{y}$ est en rouge, $B_{X}$ en bleu et $B_{Z}$ en vert.

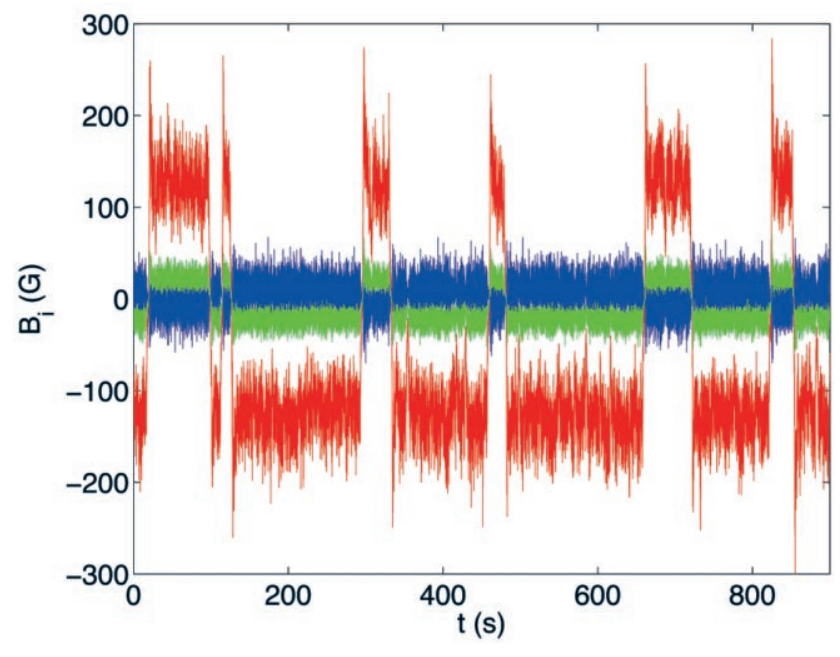

Les écoulements qui sont à l'origine des dynamos naturelles sont pour la plupart en rotation globale importante, à cause du mouvement d'ensemble de la planète ou de l'étoile. Dans nos expériences, on peut imposer une rotation de ce type en faisant tourner une turbine plus rapidement que l'autre. Nous avons alors découvert que le champ magnétique créé par l'effet dynamo, au lieu d'être statistiquement stationnaire comme lorsque les turbines tournent à la même vitesse, évolue au cours du temps avec des renversements erratiques de sa direction (fig. 3) [6]. Ce comportement, avec inversions aléatoires du champ et excursions, est très similaire à ce que l'on sait de l'évolution du champ terrestre au cours des âges. Les observations paléomagnétiques montrent en effet une alternance d'orientations Nord-Sud et Sud-Nord qui marquent les renversements du champ magnétique terrestre. Comme dans l'expérience en sodium, le temps mis pour un retournement (quelques milliers d'années pour la Terre, quelques secondes ici) est très court devant la durée moyenne d'une plage de champ magnétique de polarité donnée (quelques centaines de milliers d'années pour la Terre, quelques centaines de secondes ici).

Ce résultat de l'expérience VKS2 montre que certaines caractéristiques de la dynamo terrestre peuvent être reproduites et étudiées " au laboratoire " dans des situations bien contrôlées. De plus, la richesse des régimes observés dans l'expérience laisse entrevoir la possibilité de comprendre pourquoi des dynamos très différentes sont souvent observées pour des objets naturels a priori similaires : la Terre a un champ magnétique, Vénus n'en a pas ; notre Soleil a un cycle d'activité magnétique périodique de 22 ans [7], très particulier dans la diversité des comportements stellaires.

Les perspectives de l'expérience VKS2 concernent maintenant la recherche des ingrédients nécessaires dans l'expérience à l'effet dynamo, l'étude quantitative de la bifurcation et l'exploration des dynamiques complexes observées dans le cas où les turbines ne tournent pas à la même vitesse.

\section{- Nombres de Reynolds}

Le nombre de Reynolds cinétique (nombre sans dimension) est défini par : $R_{e}=U L / v$, où $U$ et $L$ correspondent respectivement à une vitesse et une taille caractéristiques de l'écoulement, et $v$ à la viscosité cinématique. II caractérise l'importance relative du transport de quantité de mouvement d'une part, par advection ${ }^{1}$ par le champ de vitesse $U$ et, d'autre part, par diffusion visqueuse. En général, le fluide devient turbulent au-delà d'un nombre de Reynolds critique ( $R_{e c} \sim 3000$ pour l'écoulement de von Karman). Dans l'expérience VKS2, $R_{e}$ est de l'ordre de $10^{6}$ à $10^{7}$ à comparer à $10^{8}$ pour le noyau de fer liquide dans la Terre et $10^{3}$ - $10^{4}$ dans les simulations numériques.

Le nombre de Reynolds magnétique est : $R_{m}=\mu \sigma U L$, où $\mu$ correspond à la perméabilité magnétique et $\sigma$ à la conductivité électrique du fluide. II traduit l'importance de l'advection du champ magnétique par rapport à la diffusion. On choisit le sodium malgré les problèmes de sécurité qu'il pose, car c'est le meilleur fluide conducteur de l'électricité autour de $100-150^{\circ} \mathrm{C}$. Dans l'expérience VKS2, $\mathrm{R}_{\mathrm{m}}$ augmente avec $U$ et donc avec la vitesse de rotation des turbines, jusqu'à atteindre une valeur de l'ordre de 50 à comparer à $10^{2}$ pour le noyau liquide dans la Terre.

1. Advection est le terme utilisé couramment pour parler "d'entraînement " et pour le distinguer de la convection d'origine thermique.

\section{- Induction unipolaire et effet dynamo}

(a) La rotation à vitesse angulaire $\Omega$ d'un disque conducteur soumis à un champ magnétique $\mathrm{B}_{0}$ engendre une force électromotrice proportionnelle à $\Omega$ et $B_{0}$ entre $A$ et $P$. Si l'on ferme le circuit à l'aide de balais, un courant I circule donc dans la résistance.

(b) La difficulté rencontrée par Siemens et Wheatstone, qui utilisaient des dispositifs beaucoup plus compliqués que celui de la figure, consistait à engendrer un courant sans appliquer un champ magnétique externe $B_{0}$. L'idée est de choisir la géométrie du circuit électrique afin d'utiliser le courant induit pour engendrer le champ magnétique $B$ nécessaire. On est ainsi conduit à un problème typique d'instabilité : une perturbation de champ engendre un courant qui à son tour amplifie le champ si le sens de rotation est choisi convenablement en fonction de l'induction mutuelle entre le circuit et le disque et si ce dernier tourne suffisamment vite pour compenser les pertes par effet Joule.
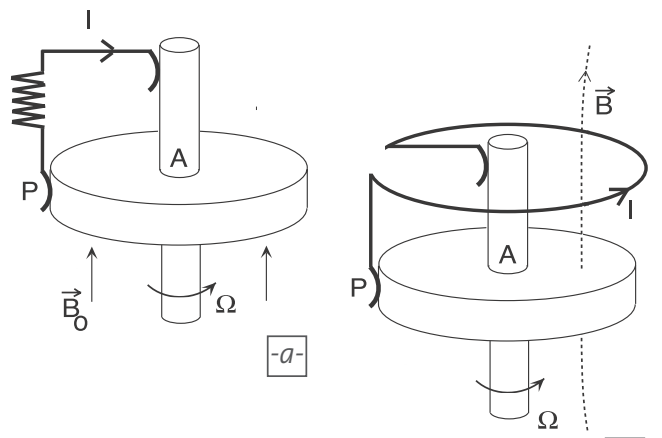